\title{
TNF-a suppresses drug resistance via NF-KB/PXR pathway in non-small cell lung cancer
}

\author{
Weiqing Huang \\ Department of Pathology \\ Ping Wang \\ Department of Pulmonary Medicine \\ Shiyu Qi \\ Department of Pathology \\ Yuanyuan Zhang \\ Department of Pathology \\ Wei Han \\ Department of Pulmonary Medicine \\ Qingnuan Kong ( $\square$ nuanyun4621@126.com ) \\ Qingdao Municipal Hospital
}

\section{Research}

Keywords: Non-small cell lung cancer, TNF-a, Multidrug resistance

Posted Date: January 20th, 2021

DOI: https://doi.org/10.21203/rs.3.rs-148624/v1

License: (c) (i) This work is licensed under a Creative Commons Attribution 4.0 International License. Read Full License 


\section{Abstract \\ Background}

Reduced sensitivity to chemotherapeutic agents caused by multidrug resistance is the main obstacle in the treatment of non-small cell lung cancer (NSCLC). Activated NF-KB/PXR pathway stimulated by procytokine TNF-a is implicated in the formation of multidrug resistance phenotype. However, the underlying mechanisms are obscure, and results concluded in various tumors are conflicted. Herein, we explored the effects of TNF-a on NFKB/PXR pathway activation and multidrug resistance development in NSCLC.

\section{Methods}

Cancerous tissues were used to evaluate the expression of TNF-a on inflammatory stroma cells and tumor cells separately and analyze their correlations with the NF-KB/PXR pathway as well as clinical chemoresistance. Alterations of the NF-KB/PXR pathway and multidrug resistance gene ABCB1 transcripts in response to TNF-a application were observed on the A549 cell line.

\section{Results}

Our results demonstrated that TNF-a produced by inflammatory stromal cells was associated with clinical chemoresistance and negatively correlated to PXR expression. Moreover, TNF-a induced activated NF-KB and repressed PXR at mRNA and protein expression levels in A549 cancer cells. Furthermore, ABCB1 transcripts changes were consistent with PXR expression.

\section{Conclusions}

Our findings suggested potentially inhibitory effects of TNF-a produced by inflammatory stroma cells on multidrug resistance in solid NSCLC through mediating NF-KB/PXR pathway activation. These results further the understanding of the roles of inflammatory cells infiltrating tumors and suggest more subtle regulation mechanisms of TNF-a that should be further explored to discover distinct targeted molecules overcoming drug resistance.

\section{Background}

Lung cancer is the leading cause of cancer-related death worldwide, and the second prevalent malignancy in males and females ${ }^{[1,2]}$. Histologically lung cancer is classified into non-small cell lung cancer (NSCLC) and small cell lung cancer (SCLC), which account approximately for $85 \%$ and $15 \%$ of all lung cancers, respectively ${ }^{[2]}$. With the advanced development of treatments, including epidermal growth factor receptor tyrosine kinase inhibitors (EGFR-TKI) and immune checkpoint inhibitors, the incidence of mortality of NSCLC has significantly decreased, and cancer-specific survival has improved $^{[3]}$. However, traditional chemotherapy and radiotherapy are still dominant therapeutic strategies for patients deficient in gene mutations or negative for immunotherapy markers (such as PD-L1). The overall outcomes for these populations remain unchanged due to chemo-treatment failure following initial circles of chemotherapy. It is generally accepted that tumor cells increase tolerance to chemotherapeutic agents and escape drug toxicity through developing multidrug resistance (MDR ${ }^{[4]}$. Apparently, targeting and impairing tumor MDR can effectively enhance sensitivity to chemotherapeutic agents, increase drug toxicity efficiency, and improve prognosis. However, the underlying molecular mechanisms involved in drug resistance acquisition are complicated and remain unclear.

Various cellular mechanisms, including aberrant apoptosis, genetic/epigenetic changes, dysregulated DNA damage repair system, and modification of drug transport, have been proposed as responsible for $\mathrm{MDR}^{[5]}$. Accumulating evidence revealed the acceleration of drug elimination caused by disordered cell membrane transporters has a crucial role in MDR phenotypes ${ }^{[5,6]}$. Furthermore, recent studies suggested that cytokines released by tumor cells and associated stromal cells accountable for dysregulation of drug efflux transporter, such as the ABCB1 gene, have a major influence in drug resistance ${ }^{[7]}$. As a pro-inflammatory cytokine, TNF- $a$ is considered a key regulator in drug resistance. It serves as a promising target for anti-drug resistance therapy ${ }^{[8-10]}$. For instance, docetaxel can induce TNF-a release by MCF-7 breast cancer cells and A2780 ovarian cancer cells ${ }^{[11]}$. TNF-a promotes HCC cell resistance to sorafenib through inducing epithelial-mesenchymal transition (EMT) ${ }^{[12]}$. Increased TNF- $a$ has been associated with tumor resistance to TKI treatment in NSCLC ${ }^{[13]}$. Thus, a better understanding of the regulatory roles of TNF- $a$ in drug resistance can shed light on identifying precise targets and drugs and improving therapeutic efficiency.

TNF-a is a 17-kDa protein containing 157 amino acids produced by inflammatory cells, including activated macrophages, $\mathrm{T}$ lymphocytes, and natural killer (NK) cells. Other cells that express lower levels of TNF- $a$ are fibroblasts, smooth muscle cells, and tumor cells ${ }^{[7]}$. TNF-a binds to two distinct receptors including TNF-a receptor 1 (TNFR1) and TNF-a receptor 2 (TNFR2) and controls multiple downstream pathways to exert biological functions including apoptosis pathways, such as Bax/Bcl-2, caspase pathway, and cell proliferation pathway such as c-fos/c-Jun, NF-KB pathway ${ }^{[14]}$. The fact that TNF- $a$ binds to TNFR1 and induces NF-KB/PXR pathway activation has been proposed as crucial regulatory mechanisms drug resistance through transcriptional activation of a variety of membranous transporters involved in expelling chemotherapy agents including ABCB1 mentioned above. 
However, regulating properties remain controversial. Previous studies have shown that TNF- $a$ can inhibit PXR transcripts in hepatic cancer ${ }^{[15]}$. In contrast, TNF- $a$ can promote PXR expression and ABCB1 activation in several breast cell lines ${ }^{[16]}$. It is postulated that TNF- $a$ involves in NF-KB/PXR pathway modulation in a tissue-specific manner. Therefore, more data are needed to further explore the underlying regulatory mechanisms in different cancer categories to discover accurate therapeutic target biomarkers for individual therapy.

To further elucidate the implications of TNF- $a$ in chemotherapy resistance of NSCLC, we evaluated the correlations of TNF- $a$ on tumor cells (TNF-TCs), TNF-a on inflammatory stroma cells (TNF-ICs), and NF-KB, and PXR expression levels in NSCLC tissues, and investigated their roles in chemotherapy resistance. NSCLC cell line was used to evaluate NF-KB/PXR pathway and downstream ABCB1 transcripts alterations in response to TNF-a inducement.

\section{Materials And Methods}

\section{Patients}

Tissue samples were obtained from 114 EGFR wt NSCLC patients who underwent lobectomy or pneumonectomy from January 2017 to November 2018 at Qingdao Municipal Hospital, including 72 men (average age 64.7, ranging from 47-81), and 42 women (average age 62.1, ranging from 4582). Histologically, 38 invasive squamous cell carcinoma (7 keratinizing type and 31 non-keratinizing type) and 76 invasive adenocacinoma ( 34 acinar predominant, 17 lepidic predominant, 14 solid predominant, 9 papillary predominant, 2 micropapillary predominant) were included in this study. Tumor staging (pTNM) was evaluated according to Pathologic Stage Classification (AJCC $8^{\text {th }}$ Edition): 63 cases were T1 ( 3 cases T1a, 40 cases T1b and 20 cases T1c), 44 cases were T2 (35 cases T2a and 9 cases T2b), 3 cases were T3, and 4 cases were T4. Lymph node metastasis occurred in 87 cases; no metastatic lymph nodes were observed in 27 cases. Ninety-two cases had distant metastasis, and 22 cases did not have distant metastasis. All patients were chemotherapy- and radiotherapy-naïve ahead of surgical resection. Of 114 cases, 107 cases received platinum-based chemotherapy for 4-6 cycles. Chemoresistance was defined as tumor recurrence occurring within 3-6 months of chemotherapy. Otherwise, chemosensitivity was designated. Written informed consent was obtained from all patients involved. The current study was approved by the Ethical Review Committee of Qingdao Municipal Hospital (Qingdao, Shandong, China)

\section{Immunohistochemistry}

The selected paraffin-embedded blocks were cut into 4-5 $\mu$ m slices. Immunohistochemistry was performed on an automatic staining system (Roche Benchmark XT, Swiss) with primary antibodies against TNF-a (1:150; Abcam, ab6671, USA), NF-kB (1:300; Abcam, ab16502, USA) and PXR (1:100; Abcam, ab85451, USA). Appropriate positive controls were used to confirm the staining efficiency (human intestinal tissue for TNF-a; colon tissue for NF-KB, and liver tissue for PXR). PBS was used as a substitution of primary antibodies for negative controls. All slides were scored by two practical pathologists blinded to the whole experiment. The total scores were measured by staining intensity ( 0 : blank; 1 : light yellow; 2 : brown-yellow; 3 : brown) multiplied by percentage of positive area (0: $<5 \% ; 1: 5 \%-25 \% ; 2: 26 \%-50 \% ; 3: 51 \%-75 \% ; 4:>75 \%)$. The sum $0-4$ was designated as low expression, and 5-12 was designated as high expression.

\section{Cell culture}

Human non-small cell lung cancer cell line (A549) and human normal bronchial epithelial cell line (BEAS-2B) were purchased from Wuhan Procell (Wuhan, China), grown in RPMI-1640 (Hyclone, USA) medium supplemented with 10\% fetal bovine serum (FBS, Hyclone, USA) and maintained at 37 ${ }^{\circ} \mathrm{C}$ in an incubator with $5 \% \mathrm{CO}_{2}$ and $95 \%$ humidity. For experimental research, the cells were incubated for $12 \mathrm{~h}$ with $10 \mathrm{ng} / \mathrm{mL}$ of recombinant human TNF-alpha (rhTNF-a) (Sino Biological, Beijing, China).

\section{RNA extraction and real-time quantitative PCR}

Total RNA was isolated using the PureLink RNA Mini Kit (Invitrogen, USA), according to the manufacturer's instruction. The concentration and purity of total RNA were determined by NanoDrop 2000 Spectrophotometer (Thermo Fisher Scientific, USA). cDNA was synthesized by reverse transcription of 1 mg total RNA using PrimeScript ${ }^{\mathrm{TM}} \mathrm{RT}$ reagent Kit (Takara, Japan), and gDNA Eraser was added to eliminate the genomic DNA contamination. The amplification was performed using a TaKaRa TB Green® Premix Ex Taq ${ }^{\text {TM }}$ II kit (Takara, Japan) on a LightCycler ${ }^{\circledR} 96$ System (Roche). The PCR primers were as follows: TNF- $a$, forward 5'-GTGACAAGCCTGTAGCCCATGTT-3' and reverse 5'-TTATCTCAGCTCCACGCCATTA-3'; NF- $K B$, forward 5'GTGGGGACTACGACCTGAATG-3' and reverse 5'-GGGGCACGATTGTCAAAGATG-3'; PXR forward 5'- AGCAATTCGCCATTACTCTCAAGT-3' and reverse 5'CTGGGTGCTGAGCATTGA-3'; MDR-1, forward 5'-GGGAGCTTAACACCCGACTTA-3' and reverse 5'-GCCAAAATCACAAGGGTTAGCTT-3'. The reaction conditions were as follows: $95^{\circ} \mathrm{C}$ for $15 \mathrm{~min}$, followed by 40 cycles of $94{ }^{\circ} \mathrm{C}$ for $15 \mathrm{~s}, 55^{\circ} \mathrm{C}$ for $30 \mathrm{~s}$, and $72{ }^{\circ} \mathrm{C}$ for $30 \mathrm{~s}$. PCR amplification efficiency was verified by melting peaks analysis. The relative expression levels of the target genes were calculated using the $2^{-\Delta \Delta \mathrm{Ct}} \mathrm{method}$.

\section{Western blot}

Cultured cells were mixed with 1mI RIPA Lysis and Extraction Buffer (Thermo, USA) containing 100mM PMSF, 25 mM Tris•HCl pH 7.6, 150 mM NaCl, $1 \%$ NP-40, $1 \%$ sodium deoxycholate, $0.1 \%$ SDS and protease inhibitor cocktail. The whole solution was then incubated on ice for $5 \mathrm{~min}$ and centrifuged at $\sim 14,000 \times \mathrm{g}$ for 15 minutes to collect supernatant for further analysis. Protein concentrations were determined by the Bio-Rad protein assay 
(BioRad). An equal amount of protein samples were electrophoresed on a 10\% SDS-PAGE gel (Invitrogen) transferred to PVDF membranes (Millipore Co, USA). The membranes were blocked with $5 \%$ (w/v) non-fat milk in TBST (Tris-buffer saline containing $0.1 \%$ Tween-20) for $1 \mathrm{~h}$ at room temperature and incubated with primary antibodies overnight at $4{ }^{\circ} \mathrm{C}$. The following antibodies were used: anti-NF-KB (P65) (1:2000, Abcam, ab108981), anti-NF-KB p65 (phospho S536) (1:10,000, Abcam, ab86299), anti- PXR antibody (1:1000, Abcam, ab85451) and anti-P-gP (1:2000, Abcam, ab226937). Membranes were then washed, and secondary antibodies were added for $1 \mathrm{~h}$ at room temperature. Intensities were normalized by a protein/ $\beta$-actin ratio as fold changes relative to the control value.

\section{Statistical analysis}

Statistical analyses were performed using SPSS 20.0 (SPSS Inc., Chicago, IL, USA), GraphPad Prism (La Jolla, CA, USA). Numerical data were analyzed by two-way ANOVA with post-Sidak's multiple. Categorical data were compared by the Chi-square test and Fisher exact test. Spearman correlation was also used to evaluate the correlations between variables. The two-sided P-values $<0.05$ were considered statistically significant.

\section{Results}

\section{TNF-TCs, TNF-ICs, NF-KB, and PXR expressions in NSCLC tissues}

First, we performed immunohistochemical staining to examine the protein expression of TNF-TCs, TNF-ICs, NF-KB, PXR in NSCLC tissues. Positive staining of TNF-a was found in the cytoplasm and positive staining of NF-KB and PXR in the nucleus and cytoplasm (Fig. 1A-D). Our results showed significantly higher expression levels of TNF-TCs (71.1\% vs. $57.0 \%)$, TNF-ICs (28\% vs. $14.9 \%)$, NF-KB (79.8\% vs. $29.8 \%)$, and PXR (38.6\% vs. $21.9 \%)$ in NSCLC tumor cells compared to corresponding normal controls. However, no difference in TNF-ICs was found between cancerous tissues and normal controls (Table 1, $p=0.009$ for TNF-TCs; $p=0.02$ for TNF-ICs; $p<0.001$ for NF-KB and PXR).

Table 1. Elevated expression of TNF-TCs, TNF-ICs, NF-KB, and PXR in NSCLC tissues in comparison to normal controls

\begin{tabular}{|c|c|c|c|c|c|c|c|c|c|c|c|c|c|c|c|c|c|}
\hline & \multirow[b]{2}{*}{ total } & \multicolumn{4}{|c|}{ TNF-TCs } & \multicolumn{4}{|c|}{ NF-KB } & \multicolumn{4}{|l|}{ PXR } & \multicolumn{4}{|c|}{ TNF-ICs } \\
\hline & & low & High & $\chi^{2}$ & $\begin{array}{l}P \\
\text { value }\end{array}$ & low & High & $x^{2}$ & $\begin{array}{l}P \\
\text { value }\end{array}$ & low & High & $\chi^{2}$ & $\begin{array}{l}\mathrm{P} \\
\text { value }\end{array}$ & low & High & $\chi^{2}$ & $\begin{array}{l}P \\
\text { value }\end{array}$ \\
\hline Cancer & 114 & 33 & 81 & 6.77 & 0.009 & 23 & 91 & 57.5 & $<0.001$ & 70 & 44 & 25.9 & $<0.001$ & 82 & 32 & 5.85 & 0.02 \\
\hline normal & 114 & 52 & 62 & & & 80 & 34 & & & 89 & 25 & & & 97 & 17 & & \\
\hline
\end{tabular}

\section{Correlations of TNF-TCs and TNF-ICs expressions with NF-KB and PXR expressions in NSCLC tissues}

Furthermore, we evaluated the correlations of TNF-TCs and TNF-ICs with NF-KB as well as PXR expression. Both TNF-TCs and TNF-ICs were positively associated with NF-KB (Fig.1E-F r $=0.23 \mathrm{p}=0.01$ for TNF-TCs vs NF-KB and $r=0.21 \mathrm{p}=0.03$ for TNF-ICs vs NF-KB). Moreover, there were significant negative correlations between TNF-ICs and PXR, while no correlation was observed between TNF-TCs and PXR (Fig. 1G-H r=-0.21 p=0.03 for TNF-ICs vs. PXR and $r=-0.0011 p=0.90$ for TNF-TCs vs. PXR). Therefore, our results suggested that TNF-a produced by inflammatory stroma cells modulates the NF-KB/PXR pathway in NSCLC.

\section{Correlations with clinicopathological features}

To examine the implications of TNF-a mediated inflammatory effects on chemotherapy, we analyzed the association of clinicopathological features including tumor stage, lymph node metastasis, distant metastasis, and chemotherapy response with the expressions of TNF-TCs, TNF-ICs, NF-KB, and PXR. In general, TNF-TCs and TNF-ICs were associated with tumor development with higher expression of TNF-TCs but lower expression of TNF-ICs in progressed tumors. However, significantly decreased expression of TNF-ICs was observed in chemo-resistance cases (Table 2, p=0.04). No correlation was found between TNF-TCs and chemotherapy. The results demonstrated TNF-ICs might promote drug resistance phenotype in NSCLC. Besides, similar to previous results, PXR showed an elevated expression among chemo-resistance cases compared to chemo-sensitive cases (Table 2, p<0.001).

Table 2. Correlations with clinicopathological features 


\begin{tabular}{|c|c|c|c|c|c|c|c|c|c|c|c|c|c|}
\hline & \multirow[b]{2}{*}{ total } & \multicolumn{3}{|c|}{ TNF-TCs } & \multicolumn{3}{|c|}{ NF-KB } & \multicolumn{3}{|c|}{ PXR } & \multicolumn{3}{|c|}{ TNF-ICs } \\
\hline & & low & High & P value & low & High & $P$ value & low & High & $P$ value & low & High & $P$ value \\
\hline I & 65 & 19 & 46 & 0.96 & 15 & 50 & 0.73 & 35 & 30 & 0.20 & 46 & 19 & 0.74 \\
\hline ॥ & 14 & 4 & 10 & & 2 & 12 & & 11 & 3 & & 10 & 4 & \\
\hline III & 13 & 3 & 10 & & 3 & 10 & & 10 & 3 & & 11 & 2 & \\
\hline IV & 22 & 7 & 15 & & 3 & 19 & & 14 & 8 & & 15 & 7 & \\
\hline $\mathrm{T} 1$ & 63 & 14 & 49 & 0.014 & 12 & 51 & 0.84 & 37 & 26 & 0.75 & 39 & 24 & 0.03 \\
\hline T2 & 44 & 19 & 25 & & 9 & 35 & & 28 & 16 & & 37 & 7 & \\
\hline T3+T4 & 7 & 0 & 7 & & 2 & 5 & & 5 & 2 & & 6 & 1 & \\
\hline NO & 87 & 27 & 60 & 0.6 & 17 & 70 & 0.82 & 50 & 37 & 0.30 & 58 & 29 & 0.04 \\
\hline N1 & 16 & 3 & 13 & & 3 & 13 & & 12 & 4 & & 13 & 3 & \\
\hline N2 & 11 & 3 & 8 & & 3 & 8 & & 8 & 3 & & 11 & 0 & \\
\hline MO & 92 & 26 & 66 & 0.81 & 21 & 71 & 0.15 & 56 & 36 & 0.81 & 69 & 23 & 0.14 \\
\hline M1 & 22 & 7 & 15 & & 2 & 20 & & 14 & 8 & & 13 & 9 & \\
\hline CS & 42 & 13 & 29 & 0.85 & 9 & 33 & 0.86 & 30 & 12 & $<0.001$ & 26 & 16 & 0.04 \\
\hline CR & 65 & 19 & 46 & & 13 & 52 & & 11 & 54 & & 52 & 13 & \\
\hline
\end{tabular}

\section{The inducing effects of TNF-a on NF-KB/PXR pathway}

To further elucidate the inducing effects of TNF-a on regulating NF-KB/PXR signaling in NSCLC tumor cells, we used an NSCLC cell line (A549) to determine mRNA and protein expression levels of PXR and NF-KB at $12 \mathrm{~h}$ after TNF- $\mathrm{a}$ exposure. BEAS-2B cell line was used as a negative control. At the baseline, the results showed a comparable mRNA expression of NF-KB between the A549 cell line and BEAS-2B cell line. NF-KB was concurrently unregulated in response to TNF-a treatment in the two cell lines (Fig. $2 A, p<0.001$ for A549 cells and $p<0.0001$ for controls). The elevation of NF-KB expression in the A549 cell line was much higher as compared to that in normal controls (Fig. 2A, $p<0.0001$ ). Western blot analysis showed that TNF-a treatment induced increased NF-KB protein expression after TNF treatment for both cell lines (Fig. 2E, p<0.001 for A549 cells and controls).

To further explore whether TNF-a treatment induces NF-KB functional activity, we also investigated the protein expression levels of phosphor-NF-kB (S536) in A549 and BEAS-2B cell line $12 \mathrm{~h}$ after TNF-a application. Our results showed that TNF-a treatment caused elevated phosphor-NF-KB in both cell lines (Fig. 2F, p<0.001 for A549 cells and controls). Also, consistent with mRNA expression changes, A549 had more abundant phosphor-NF-kB expression levels than controls after TNF-a treatment (Fig. 2F, $p<0.01)$.

TNF-a exposure resulted in reduced PXR mRNA expression in the A549 cell line (Fig. 2B, p<0.01). Conversely, the expression of PXR was markedly elevated in the control cell line (Fig. 2B, $\mathrm{p}<0.01$ ). Furthermore, the protein alterations of PXR were consistent with the changes at mRNA expression level (Fig. 2G, p<0.001 for both cell lines). To examine the transcriptional activities of PXR, we investigated mRNA and protein levels of PXR target gene ABCB1 following TNF-a treatment, and whether mRNA level or protein level alterations of ABCB1 was identical to PXR alterations (Fig. 2C, $p<0.001$ for A549 cells and $p<0.01$ for controls; Fig. $2 \mathrm{H}, \mathrm{p}<0.001$ for A549 cells and $p<0.01$ for controls).

\section{Discussion}

In this study, we used NSCLC cancerous tissues and the A549 cell line to elucidate the implications of TNF-a during the inducement of the NF-KB/PXR pathway and their correlations with chemotherapy resistance. We found that TNF-ICs was negatively associated with PXR and chemosensitivity. Moreover, we discovered that TNF-a secreted by inflammatory stromal cells had a higher effect in controlling chemotherapy resistance compared to TNF-a secreted by tumor cells. TNF-a secreted by inflammatory stromal cells predominantly affects chemotherapy resistance via regulating NF$\mathrm{KB} / \mathrm{PXR}$ pathway and PXR transcripts, which was further confirmed in vitro. TNF-a treatment on the A549 cell line triggered the upregulation and activation of NF-KB and reduced the expression levels of PXR as well as its target gene ABCB1. Our results showed that within the solid tumor of NSCLC, exogenous TNF-a secreted by stroma might lead to repressive effects on the chemotherapy resistance phenotype of NSCLC cells. Moreover, NF-KB/PXR pathway activation resulted in a key modulation mechanism involved. 
Resistance to therapeutic agents is a major factor leading to an unfavorable outcome in anti-cancer therapy. To date, accumulating evidence has suggested the importance of TNF-a in inducing drug resistance. TNF-a and MMP-9 baseline levels were found to be significantly elevated in metastatic renal-cell carcinoma resistant to sunitinib ${ }^{[17]}$. The combined application of anti-TNF- $a$ drugs demonstrated a promising therapeutic direction for improving the efficiency of chemotherapy agents, TKI, and immunotherapy. The combination of anti-TNF-a treatment and chemotherapy can suppress colon cancer cells' survival and reduce drug resistance ${ }^{[8]}$. The application of anti-TNF- $a$ drugs can also overcome resistance to anti-PD-1 in experimental melanoma in vivo and in vitro ${ }^{[9,10,18]}$. In NSCLC, TNF- $a$ blockade has been identified to enhance EGFR inhibition effectiveness ${ }^{[13]}$.

As a pro-inflammatory cytokine with biphasic functions that modulate both pro-carcinoma and anti-carcinoma downstream, anti-TNF-a therapy has potential risks. It may lead to cellular proliferation and promote malignant transformation. Anti-TNF-a can account for a mild risk of incident cancer in a population of IBD patients with recent malignancy ${ }^{[19]}$. Moreover, a previous study found that rheumatoid arthritis (RA) patients treated with anti-TNFa have an increased risk of developing non-melanoma skin cancer, especially squamous cell carcinoma ${ }^{[20]}$. Therefore, further exploration of the regulating mechanisms of TNF-a is important to enhance drug efficiency and avoid side effects.

Recent results suggested the implications of the NF-KB/PXR signaling pathway involved in TNF- $a$ inducing drug resistance. TNF- $\alpha$ affects drug resistance by modulating the NF-KB/PXR signaling pathway to control the PXR target genes' transcripts, including $A B C B 1, C Y P 3 A 11, A B C G 2$, and $\operatorname{GSTa}^{[16,17,21]}$. However, the regulating mechanisms of TNF- $a$ in the NF-KB/PXR signaling pathway lack homogeneity. DEN-induced hepatic cancer in mice resulted in increased expression of TNF- $\alpha$ and NF-KB; the expression of PXR was concurrently reduced when compared to control mice ${ }^{[16]}$. Longterm (24-96 h) TNF-a treatment disrupts NF-KB/p65 activation, reduces the nuclear accumulation of NF-KB/p65, and decreases ABCB1 expression, leading to sensitization towards drug treatment in colon cancer ${ }^{[22]}$. TNF- $a$ and IL-1 $\beta$ treatment for $72 \mathrm{~h}$ induces upregulation of $A B C G 2$ and PXR expression consistent with NF-KB activity in some breast cancers, including MCF7, BT-474, CAL51, 184A1, and HBL100 cells ${ }^{[17]}$. Our study found that TNF-a treatment causes activation of NF-KB and decreases transcripts and functions of PXR in NSCLC.

In most cancers, TNF-a binds to TNFR1 on the cellular membrane and activates NF-KB signaling in a canonical pathway, thus resulting in transcriptional activities of multiple genes ${ }^{[14,23,24]}$. It has been proved that NF-kB affects gene transcripts of PXR by interrupting the PXR-RXRa interaction. PXR is a member of orphan NRs that shares common structural domains consisting of an N-terminal activation function domain 1 (AF-1), a conserved zinc-finger-type DNA binding domain (DBD), and a C-terminal ligand-binding domain (LBD) ${ }^{[16]}$. PXR is differentially expressed in human cancers: it is increased in prostate, breast, and endometrial, and decreased in colorectal and cervical cancer ${ }^{[25,26]}$. In addition, to regulate genes involved in cellular proliferation, tumor metastasis, and apoptosis, PXR targets multidrug resistance protein 1 (MDR1, also known as ABCB1), a drug efflux transporter that encodes P-GP and serves as a major regulator of drug resistance through pumping out anti-cancer agents. Overexpression of $A B C B 1$ is associated with docetaxel and cisplatin-induced drug resistance, which is a frequent problem in the procedure of chemotherapy in NSCLC. Both docetaxel and cisplatin promote elevated expression of $A B C B 1$ in 3D-cultured NSCLC cells, albeit the latter is not a substrate of ABCB1 [27]. Thus, the decreased PXR and ABCB1 expressions in response to TNF- $a$ application indicates repressive roles of TNF- $\alpha$ in chemotherapy resistance which is consistent to the evidence deduced in histologic tissues.

The inflammatory regulator network is a bilateral and complicated process. PXR can mutually interplay with and TNF-a and NF-KB. PXR diminishes the inflammatory injury generally through the negative modulation of NF-KB and TNF-a ${ }^{[26]}$. Thus, rather than a unilateral observation, research on feedback loops is recommended to identify and provide complete information for anti-cancer treatment. The approach for a clinical application requires more detailed research.

In this study, we used an A549 cell line for detecting inducing roles of TNF-a in vitro. A549 cell line has maximal mRNA and protein expression of PXR among various NSCLC cell lines, including A549, NCl-H358, HCC827, NCl-H1650, and NCl-H1299. Our results demonstrated that despite resembling activation of NF-KB, A549 cells displayed repressed PXR expressions in response to TNF-a inducement compared to activated PXR transcripts in normal controls. The contrary alterations of PXR expression in NSCLC and normal cells suggest TNF-a has differential regulatory roles on the NFKB/PXR pathway between benign and malignancies; TNF-a treatment for drug resistance may accelerate and deteriorate malignant transformation in normal cells; however, this needs to be further explored. Furthermore, it has been known that cytokines can influence cellular proliferation, drug resistance, as well as apoptosis in both autocrine way and/or paracrine way ${ }^{[17]}$. Although our results demonstrated that TNF-ICs but not TNF-TCs had negative associations with PXR, which suggests the paracrine way is more likely to involve drug resistance, further experiments are required to determine the roles of autocrine TNF-a in mediating drug resistance of NSCLC.

In conclusion, our data revealed that TNF-a expression in tumor-infiltrating inflammatory cells is a predictive biomarker for chemotherapy drug resistance in NSCLC. NF-KB activation and PXR repression triggered by TNF-a are involved in the underlying molecular mechanism. Our findings proposed that the application of TNF-a inhibitors in anti-cancer therapy may block TNF-a secretion of ICs and improve drug resistance in NSCLC. On the other side, TNF-a treatment may sensitize chemotherapy; yet, adverse effects of cellular proliferation promotion should be taken into consideration.

Our findings further the understanding of the roles of inflammatory cells infiltrating tumors and suggest that more subtle regulation mechanisms of TNF-a should be taken into consideration in the clinical usage of TNF-a or its inhibitors. 


\section{Abbreviations}

NSCLC, non-small cell lung cancer; IBD, inflammatory bowel disease; NF-KB, Nuclear factor kappa-B; PXR, Pregnane X receptor; Bax, associated X protein; Bcl-2, B cell lymphoma 2; ABCB1, ATP-binding cassette, sub-family B, member1; EGFR, Epidermal Growth Factor Receptor; PD-L1, Programmed cell death 1 ligand 1; CYP3A11, Cytochrome P450 3A11; ABCG2, ATP-binding cassette G2; GSTa2, Glutathione S-transferase A2; TNF-a, Tumor Necrosis Factor alpha.

\section{Declarations}

\section{Ethics approval and consent to participate}

The experimental protocol was established, according to the ethical guidelines of the Helsinki Declaration and was approved by the Human Ethical Review Committee of Qingdao Municipal Hospital (Qingdao, Shandong, China). Written informed consent was obtained from individual or guardian participants.

\section{Consent for publication}

Not applicable

\section{Availability of data and materials}

The authors declare that all data supporting the findings of this study are available within the article.

\section{Competing interest}

The authors declare that they have no competing interests

\section{Funding}

This study was supported by grants from the National Natural Science Foundation of China (no.81973012) and the Qingdao Outstanding Health Professional Development Fund.

\section{Authors' Contributions}

QK and WH designed and supervised the experiments. XY, SQ, YZ performed the experiments. WH analyzed data. QK and WH wrote the manuscript. All authors read and approved the final manuscript.

\section{Acknowledgements}

Thanks for the support of Beijing DAO Research Technology Ltd (www.lingyankeyan.com) in research design, data processing and statistical analysis.

\section{References}

1. Lung Cancer Incidence and Mortality with Extended Follow-up in the National Lung Screening Trial. J Thorac Onco/2019, 14:1732-1742.

2. Schabath MB, Cote ML: Cancer Progress and Priorities: Lung Cancer. Cancer Epidemiol Biomarkers Prev 2019, 28:1563-1579.

3. Howlader N, Forjaz G, Mooradian MJ, Meza R, Kong CY, Cronin KA, Mariotto AB, Lowy DR, Feuer EJ: The Effect of Advances in Lung-Cancer Treatment on Population Mortality. N Engl J Med 2020, 383:640-649.

4. Schild SE, Tan AD, Wampfler JA, Ross HJ, Yang P, Sloan JA: A new scoring system for predicting survival in patients with non-small cell lung cancer. Cancer Med 2015, 4:1334-1343.

5. Lim ZF, Ma PC: Emerging insights of tumor heterogeneity and drug resistance mechanisms in lung cancer targeted therapy. $J$ Hematol Oncol $2019,12: 134$

6. Robey RW, Pluchino KM, Hall MD, Fojo AT, Bates SE, Gottesman MM: Revisiting the role of ABC transporters in multidrug-resistant cancer. Nat Rev Cancer 2018, 18:452-464.

7. Mercogliano MF, Bruni S, Elizalde PV, Schillaci R: Tumor Necrosis Factor a Blockade: An Opportunity to Tackle Breast Cancer. Front Oncol 2020, 10:584.

8. Li W, Xu J, Zhao J, Zhang R: Oxaliplatin and Infliximab Combination Synergizes in Inducing Colon Cancer Regression. Med Sci Monit 2017, 23:780-789.

9. Bertrand F, Montfort A, Marcheteau E, Imbert C, Gilhodes J, Filleron T, Rochaix P, Andrieu-Abadie N, Levade T, Meyer N, et al: TNFa blockade overcomes resistance to anti-PD-1 in experimental melanoma. Nat Commun 2017, 8:2256. 
10. Montfort A, Colacios C, Levade T, Andrieu-Abadie N, Meyer N, Ségui B: The TNF Paradox in Cancer Progression and Immunotherapy. Front Immunol 2019, 10:1818.

11. Edwardson DW, Boudreau J, Mapletoft J, Lanner C, Kovala AT, Parissenti AM: Inflammatory cytokine production in tumor cells upon chemotherapy drug exposure or upon selection for drug resistance. PLoS One 2017, 12:e0183662.

12. Tan W, Luo X, Li W, Zhong J, Cao J, Zhu S, Chen X, Zhou R, Shang C, Chen Y: TNF-a is a potential therapeutic target to overcome sorafenib resistance in hepatocellular carcinoma. EBioMedicine 2019, 40:446-456.

13. Gong K, Guo G, Gerber DE, Gao B, Peyton M, Huang C, Minna JD, Hatanpaa KJ, Kernstine K, Cai L, et al: TNF-driven adaptive response mediates resistance to EGFR inhibition in lung cancer. J Clin Invest 2018, 128:2500-2518.

14. van Horssen R, Ten Hagen TL, Eggermont AM: TNF-alpha in cancer treatment: molecular insights, antitumor effects, and clinical utility. Oncologist 2006, 11:397-408.

15. Kotiya D, Jaiswal B, Ghose S, Kaul R, Datta K, Tyagi RK: Role of PXR in Hepatic Cancer: Its Influences on Liver Detoxification Capacity and Cancer Progression. PLoS One 2016, 11:e0164087.

16. Malekshah OM, Lage H, Bahrami AR, Afshari JT, Behravan J: PXR and NF-KB correlate with the inducing effects of IL-1 $\beta$ and TNF-a on ABCG2 expression in breast cancer cell lines. Eur J Pharm Sci 2012, 47:474-480.

17. Jones VS, Huang RY, Chen LP, Chen ZS, Fu L, Huang RP: Cytokines in cancer drug resistance: Cues to new therapeutic strategies. Biochim Biophys Acta 2016, 1865:255-265.

18. Cervera-Carrascon V, Siurala M, Santos JM, Havunen R, Tähtinen S, Karell P, Sorsa S, Kanerva A, Hemminki A: TNFa and IL-2 armed adenoviruses enable complete responses by anti-PD-1 checkpoint blockade. Oncoimmunology 2018, 7:e1412902.

19. Poullenot F, Seksik P, Beaugerie L, Amiot A, Nachury M, Abitbol V, Stefanescu C, Reenaers C, Fumery M, Pelletier AL, et al: Risk of Incident Cancer in Inflammatory Bowel Disease Patients Starting Anti-TNF Therapy While Having Recent Malignancy. Inflamm Bowel Dis 2016, 22:1362-1369.

20. Wang JL, Yin WJ, Zhou LY, Zhou G, Liu K, Hu C, Zuo XC, Wang YF: Risk of non-melanoma skin cancer for rheumatoid arthritis patients receiving TNF antagonist: a systematic review and meta-analysis. Clin Rheumatol 2020, 39:769-778.

21. Wahli W: A gut feeling of the PXR, PPAR and NF-kappaB connection. J Intern Med 2008, 263:613-619.

22. Walther W, Kobelt D, Bauer L, Aumann J, Stein U: Chemosensitization by diverging modulation by short-term and long-term TNF-a action on ABCB1 expression and NF-KB signaling in colon cancer. Int J Oncol 2015, 47:2276-2285.

23. Gernez L, Poiret C: [Use of injectable Tranxene during labor]. Lille Med 1974, 19:863-866.

24. Taniguchi K, Karin M: NF-KB, inflammation, immunity and cancer: coming of age. Nat Rev Immunol 2018, 18:309-324.

25. Pondugula SR, Pavek P, Mani S: Pregnane X Receptor and Cancer: Context-Specificity is Key. Nucl Receptor Res $2016,3$.

26. Xing Y, Yan J, Niu Y: PXR: a center of transcriptional regulation in cancer. Acta Pharm Sin B 2020, 10:197-206.

27. Vesel M, Rapp J, Feller D, Kiss E, Jaromi L, Meggyes M, Miskei G, Duga B, Smuk G, Laszlo T, et al: ABCB1 and ABCG2 drug transporters are differentially expressed in non-small cell lung cancers (NSCLC) and expression is modified by cisplatin treatment via altered Wnt signaling. Respir Res 2017, 18:52.

\section{Figures}
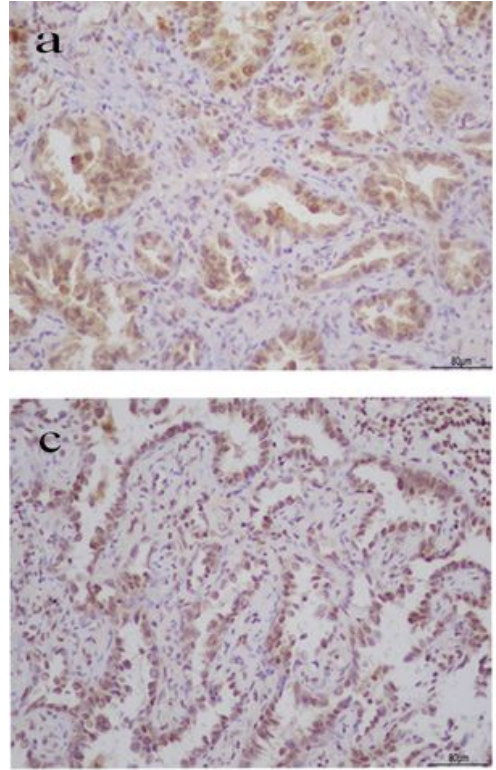
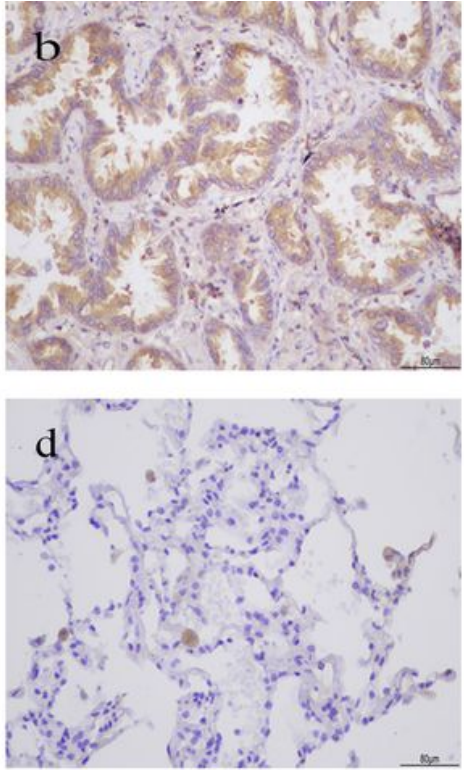
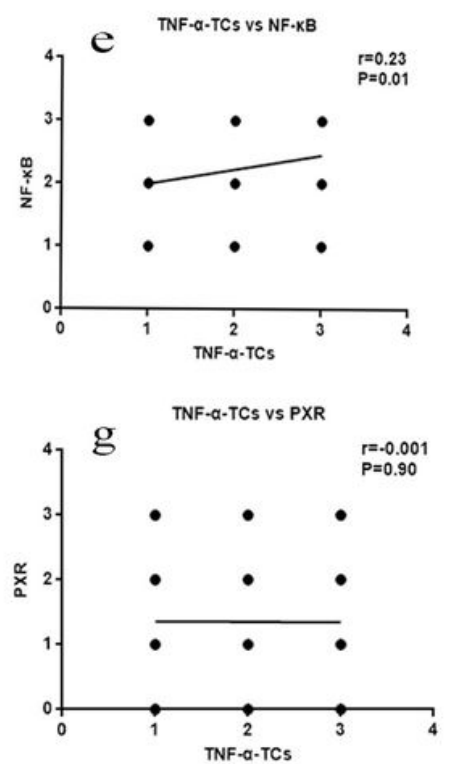
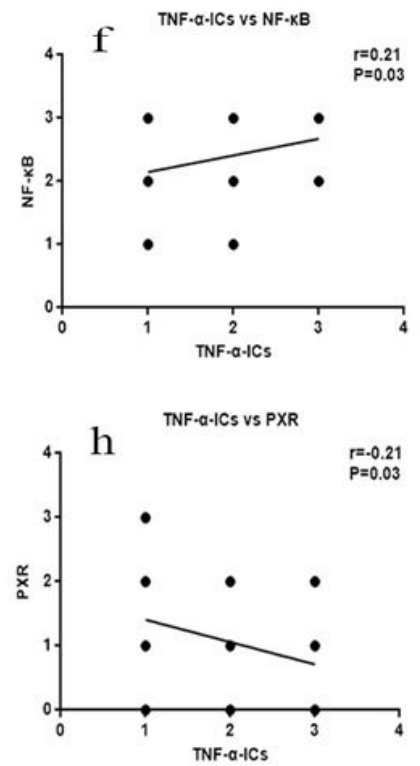
Figure 1

(a-d) Immunohistochemical staining of TNF-a (a), NF-KB (b), PXR (c), and negative control (d). e-h: Correlation analysis. (e) Association between TNF$a-T C s$ and NF-KB $(r=0.23, p=0.01)$. (f) Association between TNF-a-ICs and NF-KB $(r=0.21, p=0.03)$. (g) Association between TNF- $a-T C s$ and PXR $(r=-0.0011, p=0.90)$. (h) Association between TNF-a-ICs and PXR ( $r=-0.21, p=0.03)$. TNF- $a-T C s$, TNF-a expressed on tumor cells. TNF- $a-I C s$, TNF- $a$ expressed on inflammatory stroma cells.
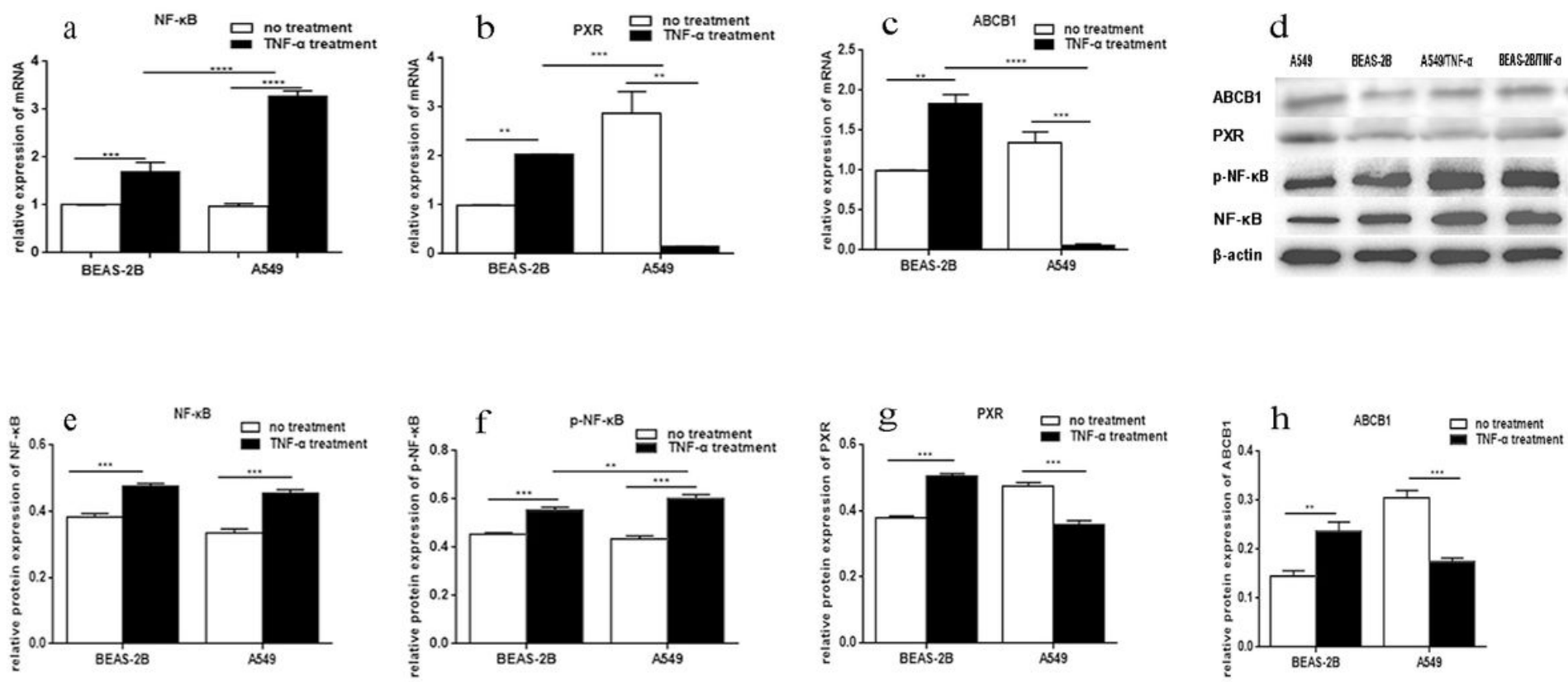

\section{Figure 2}

(a-c) Effects of TNF-a treatment on mRNA expression of NF-KB (a), PXR (b), and ABCB1 (c) after $12 \mathrm{~h} 10 \mathrm{ng} / \mathrm{mL}$ rhTNF-a treatment in A549 and BEAS2B cell line. (d-h) Immunoblot analysis of NF-KB (e), p- NF-KB (f), PXR (g), and ABCB1 (h) protein levels after $12 \mathrm{~h} 10 \mathrm{ng} / \mathrm{mL}$ rhTNF-a treatment in A549 and BEAS-2B cell line. Two-way ANOVA with post-Sidak's multiple comparisons test. $* P<0.05, * * P<0.01$, and $* * * P<0.001$, $* * * * P<0.0001$ means significant difference between compared groups. All data are presented as means \pm SEM. rhTNF, recombinant human TNF-alpha 